\title{
Risk Factor Analysis of Extended Opioid Use after Coronary Artery Bypass Grafting: A Clinical Data Warehouse-Based Study
}

\author{
Jiwon Kang ${ }^{1}$, Jae Hun Kim ${ }^{1}$, Kyung Hyun Lee ${ }^{1}$, Woo Seok Lee ${ }^{1}$, Hyoung Woo Chang ${ }^{2}$, \\ Jun Sung Kim², Kay-Hyun Park ${ }^{2}$, Cheong Lim ${ }^{2}$ \\ 'Department of Education and Training, Seoul National University Hospital, Seoul, Korea \\ ${ }^{2}$ Department of Thoracic and Cardiovascular Surgery, Seoul National University Bundang Hospital, Seongnam, Korea
}

Objectives: A clinical data warehouse (CDW) is part of our hospital information system, and it provides user-friendly 'data search and extraction' interfaces for query composition. We carried out a risk factor analysis for the extended use of opioids after coronary artery bypass grafting (CABG), taking advantage of the CDW system. Methods: From 2015 to 2017, clinical data from 461 patients who had undergone either isolated or concomitant CABG were extracted using the CDW; the extracted data included baseline patient characteristics, various examination results, and opioid prescription information. Supplementary data that could not be extracted with the CDW were collected via manual review of the electronic medical records. Results: Data from a total of 447 patients were analyzed finally. The mean patient age was $66.8 \pm 10.9$ years, 332 patients (74\%) were male, and 235 patients (53\%) had diabetes. Among the 447 patients, 90 patients (20.1\%) took some type of opioid at the 15th postoperative day. An oral rapid-acting agent was the most frequently used opioid (83\%). In the risk factor analysis for extended opioid use, duration of operation was the only significant risk factor (odds ratio $=1.004 ; 95 \%$ confidence interval, 1.001-1.007; $p=0.008$ ). Conclusions: Longer operation time was associated with the risk of extended opioid use after CABG. CDW was a helpful tool for extracting mass clinical data rapidly, but to maximize its utility, the data should be checked carefully as they are entered in the system so that post-processing can be minimized. Further refinement of the clinical data input and output interface is warranted.

Keywords: Coronary Artery Bypass, Opioid, Pain, Database Management Systems, Data Warehousing

Submitted: February 14, 2019

Revised: April 11, 2019

Accepted: April 19, 2019

\section{Corresponding Author}

Cheong Lim

Department of Thoracic and Cardiovascular Surgery, Seoul National University Bundang Hospital, Seoul National University College of Medicine, 82 Gumi-ro 173beon-gil, Bundang-gu, Seongnam 13620, Korea. Tel: +82-31-787-7134, E-mail: mluemoon@snubh.org (https:/l orcid.org/0000-0003-0913-7014)

This study was presented at the 2018 Fall Conference of the Korean Society of Medical Informatics in Chonbuk National University Hospital, Jeonju, Korea on November 23, 2018.

This is an Open Access article distributed under the terms of the Creative Commons Attribution Non-Commercial License (http://creativecommons.org/licenses/by-nc/4.0/) which permits unrestricted non-commercial use, distribution, and reproduction in any medium, provided the original work is properly cited.

(C) 2019 The Korean Society of Medical Informatics 


\section{Introduction}

Coronary artery bypass graft surgery (CABG) is an effective and commonly performed procedure for treating coronary artery disease. However, even after successful CABG, some patients suffer from postoperative pain that requires analgesics. Even 1 year after the operation, up to $17 \%$ of patients experience some degree of persistent post-sternotomy pain at rest, and the percentage increases to more than $30 \%$ upon movement [1].

Opioids are increasingly being prescribed for the treatment of diverse chronic painful conditions. Opioid use for noncancer pain management gradually increased from $24 \%$ in 2010 to $40 \%$ in 2013 in Korea [2]. Cardiac surgery and sternotomy are procedures accompanied by substantial postoperative pain, which is challenging to treat. Thus, opioids are widely used after cardiac surgery. Their use for acute pain is well accepted; however, their role in long-term treatment of chronic non-cancer pain is controversial. Prolonged use of opioids may result in adverse consequences, including tolerance, hyperalgesia, hormonal effect, and immunosuppression. Therefore, longer-term use of opioids should be carefully followed, and it is usually not recommended [2].

Meanwhile, vast quantities of data are generated every day in clinical institutes. Fortunately, the introduction of computer-based electrical medical recording enables the efficient management of data. From the past, clinical data is accumulated and renewed every day, and a clinical database warehouse $(\mathrm{CDW})$ is a computer-based electrical storage for long-term, massive information about patients. Analyzing relationships and patterns within these 'hidden' data from the CDW are expected to provide new medical insights [3].

Once a well-organized CDW is established, not only we can develop meaningful clinical outcomes by analyzing massive amount of data but also the manual work is greatly reduced.

There are a number of examples of CDW application. Sahut d'Izarn et al. [4] used their CDW to perform a study to identify the risk factors of unexpected pulmonary embolism (UPE) in cancer patients. They simply extracted the appropriate study population by previously stored diagnostic codes from the CDW rather than reviewing all medical records. Nishida et al. [5] conducted a study comparing the effects of olmesartan and candesartan monotherapy in lipid metabolism and preserving renal function. Data were retrieved from Nihon University School of Medicine (NUSM) CDW where prescription data from over 0.5 million patients are linked to their clinical information, such as demographics and diagnosis. Kassin et al. [6] used their institutional CDW to identify the risk factors of unplanned 30-day readmission after general surgery. In that work, 135 variables from 1,442 patients were drawn from a CDW, and those variables underwent univariate and multivariate analysis. Edlund et al. [7] conducted a risk factor analysis for opioid abuse/dependence for chronic non-cancer pain (CNCP) with 15,160 patients. All data were retrieved from South Central Veterans Affairs (VA) Health Care Network (VISN16) data warehouse. Grams et al. [8] studied 3.6 million US veterans to investigate risk factors for postoperative acute kidney injury (AKI). All data were retrospectively collected from National VA Corporate Data Warehouse LabChem Data.

This study also used the data collection of our institutional CDW to identify variables related to long-term use of opioids after CABG. We hope that identifying these variables will help to relieve postoperative pain and decrease longterm opioid use, resulting in a better quality of life for patients.

\section{Methods}

\section{Patients}

This was a retrospective cohort study, which was approved by the Institutional Review Board (IRB No. B-1503/290108). Patient consent was waived due the retrospective nature of the study. From the CDW of Seoul National University Bundang Hospital, we were able to retrieve data from a total of 461 patients recorded to have undergone CABG between January 2015 and December 2017. After reviewing their medical records, we excluded patients if they had expired before the 15th postoperative day $(n=10)$, if they had used opioids for reasons other than postoperative pain control $(\mathrm{n}=3)$, or if they had actually undergone other surgery instead of CABG (incorrect record) $(n=1)$.

The demographics of the patients, history of diabetes and hypertension, smoking history, marital status, education level, and preoperative left ventricular ejection fraction (LVEF) were retrieved from the CDW. To obtain details that could not be easily collected through the CDW, we reviewed the medical records of each patient. The demographics as well as the preoperative and intraoperative clinical variables of the patients who had undergone CABG are summarized in Tables 1 and 2.

The primary outcome measure was the presence of opioid use due to postoperative pain after the 15th postoperative day. Opioid usage was assessed by prescription records of opioids. If the discharged patients had received their last opioid prescription at the outpatient clinic on the 11th to 14th 
postoperative day, and the next appointment had been made a month later, we also regarded it as opioid use after the 15th postoperative day.

\section{Clinical Data Warehouse}

A CDW is part of our hospital information system, and it was designed to help users retrieve, extract, and analyze clinical information. The CDW carries all data from the hospital information system, from the start (April 2003) to a day before the current date. Because a CDW interface uses plain language with pull-down or radio button menus, researchers do not need to learn a query language. A CDW is equipped with free-text search and scenario-based retrieval of electronic medical record data [9]. The authority to use CDW is granted only to faculty members by a medical information center. With CDW, the users can draw data without the help of an information center. In this study, we composed the search conditions independently.

Table 1. Baseline characteristics of the patients $(n=447)$

\begin{tabular}{|c|c|c|}
\hline Variable & $n$ & Value \\
\hline Sex, male & 440 & $327(74)$ \\
\hline Age (yr) & 440 & $66.8 \pm 10.8$ \\
\hline Diabetes mellitus & 440 & $230(52)$ \\
\hline Hypertension & 440 & $326(74)$ \\
\hline Diagnosis & 440 & \\
\hline Silent ischemia & & $45(10)$ \\
\hline Stable angina & & $61(14)$ \\
\hline Unstable angina & & $225(51)$ \\
\hline NSTEMI & & $19(4)$ \\
\hline STEMI & & $90(21)$ \\
\hline Current smoker & 426 & $98(23)$ \\
\hline Ever married & 392 & $362(92)$ \\
\hline Level of education & 393 & \\
\hline Elementary school or none & & 75 (19) \\
\hline Middle school & & $58(15)$ \\
\hline High school & & $121(31)$ \\
\hline University & & $114(29)$ \\
\hline Graduate school or more & & $25(6)$ \\
\hline BMI $\left(\mathrm{kg} / \mathrm{m}^{2}\right)$ & 437 & $24.4 \pm 3.1$ \\
\hline Preoperative LVEF (\%) & 439 & $53.4 \pm 13.7$ \\
\hline
\end{tabular}

Values are presented as number of patients (\%) or mean \pm standard deviation.

BMI: body mass index, LVEF: left ventricular ejection fraction.

\section{Statistical Analysis}

Continuous variables were expressed as mean \pm standard deviation, and categorical variables were presented as number (\%). To identify potentially significant predictors of opioid use after the 15th postoperative day, binary logistic regression was used. Variables chosen in the univariate analysis $(p<$ 0.2 ) were included in the multivariable analysis to evaluate their significance as independent predictors. A $p$-value less than 0.05 was considered to indicate statistical significance.

Since minimally invasive direct coronary artery bypass grafting (MIDCAB) is a type of off-pump CABG, we did not include MIDCAB as a variable at this step. Instead, we performed a subgroup analysis with 307 patients who had undergone off-pump CABG by the same procedure. This time, MIDCAB was included as a variable. For statistical analyses, we used IBM SPSS version 22.0 (IBM Corp., Armonk, NY, USA).

\section{Results}

\section{Patient Characteristics and Operative Data}

The patients' baseline characteristics are shown in Table 1 . Medical records of 447 patients were reviewed. The mean age of the patients was $66.8 \pm 10.8$ years, 327 patients $(74 \%)$ were male, and 230 patients (52\%) had diabetes.

Table 2. Operative data $(n=447)$

\begin{tabular}{lc}
\hline \multicolumn{1}{c}{ Variable } & Value \\
\hline Type of surgery & $392(87.7)$ \\
CABG alone & $55(12.3)$ \\
CABG and concomitant operation & \\
Type of CABG & $307(68.6)$ \\
Off-pump & $61(13.6)$ \\
MIDCAB & $246(55.0)$ \\
Non-MIDCAB & $140(31.3)$ \\
On-pump & \\
Artery used & $112(25.1)$ \\
Unilateral ITA & $335(74.9)$ \\
Bilateral ITA & $271.5 \pm 80.6$ \\
Duration of surgery (min) & $4(0.9)$ \\
Redo open-heart surgery & \\
\hline
\end{tabular}

Values are presented as number of patients (\%) or mean \pm standard deviation.

CABG: coronary artery bypass grafting, MIDCAB: minimally invasive direct coronary artery bypass grafting, ITA: internal thoracic artery. 
Operative data are shown in Table 2. Among 447 operations, 307 cases $(68.6 \%)$ underwent off-pump CABG, including 61 cases (13.6\%) of MIDCAB. Bilateral internal thoracic arteries (ITA) were used in 335 cases (74.9\%) which is three times more often than unilateral ITA, and the mean total operative time was $271.5 \pm 80.6$ minutes.

\section{Opioid Use}

The number of patients who reported using any type of opioid for more than 15 days due to postoperative pain are shown in Table 3. Among 447 patients, 90 patients (20.1\%) reported using opioid 15 days after undergoing cardiac surgery. A rapid-acting opioid agent (IRcodon; UNIMED Co.

Table 3. Proportion of patients who used opioid for postoperative pain up to 365 days after surgery $(n=447)$

\begin{tabular}{cc}
\hline Time after surgery (day) & $\mathbf{n}(\%)$ \\
\hline 15 & $90(20.1)$ \\
30 & $35(7.8)$ \\
90 & $14(3.1)$ \\
180 & $9(2.0)$ \\
365 & $4(0.9)$ \\
\hline
\end{tabular}

Ltd., Seoul, Korea) tablet was the most frequently prescribed type of opioid (83\%). Types of opioid used are shown in Table 4.

\section{Risk Factor Analysis}

In the univariate analysis, there were five variables that had a

Table 4. Proportion of each type of opioid used after surgery $(n=90)$

\begin{tabular}{|c|c|}
\hline Type of opioid & n (\%) \\
\hline IRcodon tab. & $75(83.3)$ \\
\hline Targin CR tab. & $4(4.4)$ \\
\hline $\begin{array}{l}\text { Fentamax }^{\mathrm{c})} \text { or Durogesic } \\
\text { D-TRANS patch }\end{array}$ & $3(3.3)$ \\
\hline Oxycontin CR tab. & $2(2.2)$ \\
\hline $\begin{array}{l}\text { IRcodon tab. \& Fentamax or } \\
\text { Durogesic D-TRANS patch }\end{array}$ & $4(4.4)$ \\
\hline IRcodon tab. \& Targin CR tab. & $1(1.1)$ \\
\hline IRcodon tab. \& Oxycontin CR tab. & $1(1.1)$ \\
\hline \multicolumn{2}{|c|}{$\begin{array}{l}\text { a) IRcodon 5-mg tab. (oxycodone } \mathrm{HCl} 5 \mathrm{mg} \text { ), }{ }^{\mathrm{b})} \text { Targin CR 10/5 } \\
\text { mg tab. (oxycodone/naloxone), }{ }^{\mathrm{c})} \text { Fentamax MAT } 25 \mathrm{mcg} / \mathrm{hr} \\
\text { patch (fentanyl), }{ }^{\mathrm{d})} \text { Durogesic D-TRANS patch } 12 \mathrm{mcg} / \mathrm{hr} \text { patch } \\
\text { (fentanyl), }{ }^{\text {e) }} \text { Oxycontin CR } 10 \mathrm{mg} \text { tab. (oxycodone). }\end{array}$} \\
\hline
\end{tabular}

Table 5. Multivariable analysis for outcomes of opioid use up to 15 days after surgery $(n=447)$

\begin{tabular}{|c|c|c|c|}
\hline \multirow{2}{*}{ Variable } & \multicolumn{2}{|c|}{$p$-value } & \multirow{2}{*}{ OR $(95 \% \mathrm{Cl})$} \\
\hline & Univariate analysis & Multivariable model & \\
\hline Age & 0.280 & & \\
\hline Sex, female & 0.443 & & \\
\hline BMI & 0.534 & & \\
\hline Diabetes & 0.526 & & \\
\hline Hypertension & 0.316 & & \\
\hline Current smoker & 0.959 & & \\
\hline Ever married & 0.279 & & \\
\hline Higher education level & 0.625 & & \\
\hline Diagnosis $^{\text {a) }}$ & 0.217 & & \\
\hline On-pump CABG & 0.048 & 0.436 & \\
\hline Concomitant operation & 0.036 & 0.378 & \\
\hline Redo open-heart surgery & 0.166 & 0.167 & \\
\hline Bilateral ITA use & 0.335 & & \\
\hline Duration of surgery & 0.008 & 0.008 & $1.004(1.001-1.007)$ \\
\hline Preoperative LVEF & 0.065 & 0.170 & \\
\hline
\end{tabular}

Variables that showed $p$-values less than 0.2 in univariate analysis were included in the multivariable analysis.

OR: odds ratio, CI: confidence interval, BMI: body mass index, CABG: coronary artery bypass grafting, ITA: internal thoracic artery, LVEF: left ventricular ejection fraction.

${ }^{a)}$ Diagnosis is from silent ischemia (0) to ST elevation myocardial infarction (4), as shown in Table 1. 
p-value less than 0.2: on-pump CABG, concomitant operation, redo open heart surgery, duration of surgery, and preoperative LVEF (Table 5). These five variables were included in multivariable analyses for composite outcome. As a result, duration of surgery $(p=0.008$; odds ratio $=1.004 ; 95 \%$ confidence interval, 1.001-1.007) was shown as the only significant independent risk factor of using opioids after the 15th postoperative day.

In subgroup analysis concerning patients who underwent off-pump CABG, there were three variables that had a pvalue less than 0.2: diagnosis, redo open heart surgery, and preoperative LVEF (Table 6). None of these was proved to be an independent risk factor. In particular, MIDCAB had no significant association with the prolonged use of opioids after surgery.

\section{Discussion}

In this study, we retrospectively reviewed the current trend in opioid usage after CABG and analyzed possible factors that may result in long-term usage of opioids. As the study deals with CABG done in a single institute (Seoul National University Bundang Hospital) where three highly experienced surgeons perform all cardiac surgeries with a highly standardized surgical procedure, we could minimize operation-related bias originating from the variation of procedure by multiple operators. Additionally, unlike previous retrospective studies that retrieved data mostly by reviewing individual medical records respectively, by using CDW, it has become possible to reduce manual work excessively for retrospective research. Furthermore, instead of using subjective measures of pain (ex. VAS score), we assessed postoperative pain using prescription orders of opioids which may be objective. Thus, it was possible to overcome the limitations of studies concerning the measurement of pain. In the risk factor analysis, our data showed a statistically significant association between the duration of surgery and the presence of opioid use after the 15th postoperative day. Factors, especially those related to the invasiveness of surgery, such as bilateral ITA use, MIDCAB showed no significant difference. This result emphasizes the importance of reducing operation time in CABG for postoperative well-being of patients.

During open-heart surgery, great force is applied to the sternum and surrounding tissues by a sternum retractor to keep the thoracic cavity wide open. As the sternum remains retracted longer, surrounding tissues and nerves become

Table 6. Multivariable analysis for outcomes of opioid use up to 15 days after OPCAB $(n=307)$

\begin{tabular}{|c|c|c|c|}
\hline \multirow{2}{*}{ Variable } & \multicolumn{2}{|c|}{$p$-value } & \multirow{2}{*}{ OR $(95 \% \mathrm{Cl})$} \\
\hline & Univariate analysis & Multivariable model & \\
\hline Age & 0.667 & & \\
\hline Sex, female & 0.961 & & \\
\hline BMI & 0.935 & & \\
\hline Diabetes & 0.640 & & \\
\hline Hypertension & 0.886 & & \\
\hline Current smoker & 0.748 & & \\
\hline Ever married & 0.561 & & \\
\hline Education & 0.383 & & \\
\hline Diagnosis $^{\text {a) }}$ & 0.174 & 0.054 & $1.42(1.00-2.03)$ \\
\hline MIDCAB & 0.337 & 0.418 & $0.71(0.31-1.63)$ \\
\hline Concomitant operation & 0.529 & & \\
\hline Redo open-heart surgery & 0.066 & 0.119 & $7.45(0.60-92.70)$ \\
\hline Bilateral ITA use & 0.845 & & \\
\hline Duration of surgery & 0.819 & & \\
\hline Preoperative LVEF & 0.151 & 0.985 & $0.99(0.96-1.01)$ \\
\hline
\end{tabular}

Variables that showed $p$-values less than 0.2 in univariate analysis were included in the multivariable analysis.

OPCAB: off-pump coronary artery bypass graft surgery, OR: odds ratio, CI: confidence interval, BMI: body mass index, MIDCAB: minimally invasive direct coronary artery bypass grafting, ITA: internal thoracic artery, LVEF: left ventricular ejection fraction.

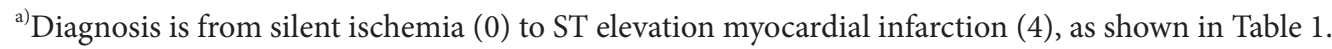


more prone to injury. Since peripheral nerve and surrounding tissue injury are known to be an important factor in the development of persistent postoperative pain [10-12], it is tempting to speculate that the tissues and nerves of patients who undergo longer surgery get more damaged, which may increase the risk of long-lasting postoperative pain. This finding was consistent with the reviews of the existing literature on non-cardiac surgery $[13,14]$. However, in the field of cardiac surgery, we could not find previous studies with related findings except one, which reported the opposite correlation between the duration of surgery and postoperative pain [15]. Though both studies are about long-lasting postoperative pain, our study set the endpoint as opioid use for pain persisting after the 15th postoperative day, while in the other study, the scale of self-reported pain lasted more than 3 months. This may explain the inconsistency between our study and the previous study.

In a subgroup analysis, interestingly, MIDCAB surgery showed no significant difference in long-term opioid usage. The result is not consistent with previous studies that have claimed the superiority of MIDCAB as leading to less chronic pain than conventional CABG. There is debate about whether MIDCAB is associated with less severe postoperative pain. There was a report that patients after MIDCAB suffered more pain during 2 days after surgery than those after conventional approach [16]. However, in that article, pain expressed by patients after MIDCAB was lesser from postoperative 3rd day and on. In our data, small sample size could account for the insignificant difference in pain with regard to MIDCAB.

This study had limitations, mostly because the data were retrieved retrospectively from the CDW. Additionally, we found a few mismatches between the CDW and individual medical records. Thus, manual correction was needed by comparing data from the CDW with individual medical records. Furthermore, we relied on records of prescription orders in determining the opioid usage after the 15th postoperative day. Prescription requiring confirmation (PRC) orders are routinely made regardless of actual pain when patients are hospitalized after surgery. Therefore, it may be difficult to confirm a relationship between PRC orders with postoperative pain. Finally, this study may not be generalized because the data in this study was limited to our single institute.

About $20 \%$ of patients who underwent CABG reported using some type of opioid due to postoperative pain for more than 15 days. Among various factors analyzed, operation time alone showed strong correlation with long-term us- age of opioid. Therefore, avoiding situations that result in extended operation time in CABG may be beneficial in decreasing postoperative pain, extended opioid use and related side effects, thus increasing overall quality of life.

This study showed that CDW is a valuable data sorting and acquisition tool, but also showed a number of innate limitations of CDW. The user interface of the electronic medical record system needs further refinements to prevent data inconsistency.

\section{Conflict of Interest}

No potential conflict of interest relevant to this article was reported.

\section{Acknowledgments}

This study was supported by a grant from Seoul National University Bundang Hospital research fund (No. 06-2015042).

\section{ORCID}

Jiwon Kang (http://orcid.org/0000-0002-0844-0284) Jae Hun Kim (http://orcid.org/0000-0002-9589-6457) Kyung Hyun Lee (http://orcid.org/0000-0001-5225-4377) Woo Seok Lee (http://orcid.org/0000-0002-5771-6443) Hyoung Woo Chang (http://orcid.org/0000-0001-5516-0333) Jun Sung Kim (http://orcid.org/0000-0003-4285-8200) Kay-Hyun Park (http://orcid.org/0000-0002-1111-9627) Cheong Lim (http://orcid.org/0000-0003-0913-7014)

\section{References}

1. Lahtinen P, Kokki H, Hynynen M. Pain after cardiac surgery: a prospective cohort study of 1-year incidence and intensity. Anesthesiology 2006;105(4):794-800.

2. Choi HY, Lee EK. Market analysis of narcotic analgesics in Korea using HIRA claims data. J Korean Acad Manag Care Pharm 2015;4(1):31-7.

3. Prather JC, Lobach DF, Goodwin LK, Hales JW, Hage ML, Hammond WE. Medical data mining: knowledge discovery in a clinical data warehouse. Proc AMIA Annu Fall Symp 1997;1997:101-5.

4. Sahut D'Izarn M, Caumont Prim A, Planquette B, Revel MP, Avillach P, Chatellier G, et al. Risk factors and clinical outcome of unsuspected pulmonary embolism in cancer patients: a case-control study. J Thromb Haemost 
2012;10(10):2032-8.

5. Nishida Y, Takahashi Y, Nakayama T, Soma M, Asai S. Comparative effect of olmesartan and candesartan on lipid metabolism and renal function in patients with hypertension: a retrospective observational study. Cardiovasc Diabetol 2011;10:74.

6. Kassin MT, Owen RM, Perez SD, Leeds I, Cox JC, Schnier $\mathrm{K}$, et al. Risk factors for 30-day hospital readmission among general surgery patients. J Am Coll Surg 2012;215(3):322-30.

7. Edlund MJ, Steffick D, Hudson T, Harris KM, Sullivan M. Risk factors for clinically recognized opioid abuse and dependence among veterans using opioids for chronic non-cancer pain. Pain 2007;129(3):355-62.

8. Grams ME, Sang Y, Coresh J, Ballew S, Matsushita K, Molnar MZ, et al. Acute kidney injury after major surgery: a retrospective analysis of veterans health administration data. Am J Kidney Dis 2016;67(6):872-80.

9. Yoo S, Hwang H, Jheon S. Hospital information systems: experience at the fully digitized Seoul National University Bundang Hospital. J Thorac Dis 2016;8(Suppl 8):S637-41.

10. Kehlet H, Jensen TS, Woolf CJ. Persistent postsurgi- cal pain: risk factors and prevention. Lancet 2006; 367(9522):1618-25.

11. Wu CL, Raja SN. Treatment of acute postoperative pain. Lancet 2011;377(9784):2215-25.

12. Alston RP, Pechon P. Dysaesthesia associated with sternotomy for heart surgery. Br J Anaesth 2005;95(2):153-8.

13. Peters ML, Sommer M, de Rijke JM, Kessels F, Heineman E, Patijn J, et al. Somatic and psychologic predictors of long-term unfavorable outcome after surgical intervention. Ann Surg 2007;245:487-94.

14. Ip HY, Abrishami A, Peng PW, Wong J, Chung F. Predictors of postoperative pain and analgesic consumption: a qualitative systematic review. Anesthesiology 2009;111(3):657-77.

15. Choiniere M, Watt-Watson J, Victor JC, Baskett RJ, Bussieres JS, Carrier M, et al. Prevalence of and risk factors for persistent postoperative nonanginal pain after cardiac surgery: a 2-year prospective multicentre study. CMAJ 2014;186(7):E213-23.

16. Walther T, Falk V, Metz S, Diegeler A, Battellini R, Autschbach R, et al. Pain and quality of life after minimally invasive versus conventional cardiac surgery. Ann Thorac Surg 1999;67(6):1643-7. 